
(25

\title{
Tobramycin adaptation enhances policing of social cheaters in Pseudomonas aeruginosa
}

Rhea G. Abisado ${ }^{\mathrm{a}}$, John H. Kimbrough ${ }^{\mathrm{ac}}$, Brielle M. Mckee ${ }^{\mathrm{a}}$, Vaughn D. Craddock ${ }^{\mathrm{a}}$, Nicole E. Smalley $^{\mathrm{b}}$, Ajai A. Dandekar ${ }^{\mathrm{b}}$, Josephine R. Chandler ${ }^{\mathrm{a} \#}$

${ }^{a}$ Department of Molecular Biosciences, University of Kansas, Lawrence, KS 66045, ${ }^{\mathrm{b}}$ University of Washington School of Medicine, Seattle, WA 98195

Running title: Tobramycin, social cheating, and policing

\#To whom correspondence should be addressed:

Josephine R. Chandler

1200 Sunnyside Ave.

Lawrence, KS 66045

Tel. 785-864-5329

Fax 785-864-5294

Email: jrchandler@ku.edu

${ }^{\mathrm{c}}$ Current address:

Department of Microbiology and Immunology

University of Iowa

51 Newton Rd

Iowa City, IA 52242

The authors declare no competing interests 27 


\section{ABSTRACT (200 max)}

The Pseudomonas aeruginosa LasR-I quorum sensing (QS) system regulates secreted proteases that can be exploited by cheaters, such as QS receptor-defective (lasR) mutants. lasR mutants emerge in populations growing on casein as a sole source of carbon and energy and increase in the population because they do not incur the substantial cost of engaging in QS. QS also increases resistance to some antibiotics, such as tobramycin. Here, we show that tobramycin suppresses the emergence of lasR mutants in casein-passaged populations. We also identify

41 several mutations that accumulate in those populations indicating evidence of antibiotic

42 adaptation. Mutations in one gene, $p t s P$, increase activity of the LasR-I system and production of

43 a QS-controlled phenazine, pyocyanin. We find that mutations in $p t s P$ lead to suppression of 44 cheaters independent of tobramycin. Cheater suppression relies on pyocyanin, which acts as a policing toxin by targeting cheaters. These results show that tobramycin suppresses las $R$ mutants through two mechanisms: first, by directly acting on tobramycin-susceptible cheaters and second, by selecting mutations in ptsP that lead to pyocyanin-dependent policing. This work demonstrates how adaptive mutations can alter the dynamics of cooperator-cheater relationships, which might be important for populations adapting to antibiotics during infections. 


\section{INTRODUCTION}

Many Proteobacteria have quorum-sensing (QS) systems that sense and respond to $\mathrm{N}$ acyl-homoserine lactones (AHLs) to cause population density-dependent changes in gene expression (1-3). AHL systems involve a LuxR-family signal receptor and a LuxI-family signal synthase $(1,4,5)$. LuxR-I systems in many Proteobacteria control production of exoproducts such as proteases and toxins, which can be considered public goods $(3,6,7)$. Public goods can be used by any member of the population; however, some population members do not contribute to public good production and are called freeloaders or cheaters (7-9). An example of a cheater is individuals with null mutations in the gene coding for the LuxR-family signal receptor; these mutants do not produce QS-dependent public goods but can still exploit public goods produced by QS cooperators in the population (8-10). Because public goods are metabolically costly, cheaters can overrun cooperators $(8,9,11)$. In conditions where the public goods are required for growth, uncontrolled cheating can lead to population collapse $(7,12-14)$. Mechanisms must exist to control cheating in order for

72 cooperative phenotypes to be maintained. One such mechanism is policing $(15,16)$. In bacteria, cooperators police cheaters by linking toxin and toxin-resistance factor production, such as through co-regulation of each of these by QS (16-18). QS can also control cheating through co- 
by QS-defective cheaters. However, these cheaters are suppressed when tetracycline is included

81 in the casein medium, because they do not express the efflux pump conferring resistance (10).

82 This type of cheater suppression likely requires some other selective force ensuring that public

83 and private goods are maintained under co-regulation (19).

In this study, we sought to determine if antibiotics can restrain the emergence of QS-

defective cheaters in bacteria other than $C$. subtsugae. We were also interested in understanding how adaptation under antibiotic selection can alter the dynamics of cooperation and cheating.

Previous reports show that AHL QS regulates antibiotic resistance in Pseudomonas aeruginosa

88 (20-23). There are two AHL QS systems in P. aeruginosa, the LasR-I and RhlR-I systems. These

two systems produce and respond to the signals 3-oxododecanoyl-homoserine lactone (3OC12-

HSL) and butanoyl-homoserine lactone (C4-HSL), respectively (3, 24, 25). The systems are hierarchical with LasR-I controlling activation of RhlR-I (25, 26). In biofilm conditions, deletions in LasR were previously shown to cause sensitivity to tobramycin antibiotic in at least one strain of $P$. aeruginosa, PAO1(20-22).

Here, we show the LasR-I system increases tobramycin resistance in planktonic

95 conditions in the $P$. aeruginosa strain PA14. We also show that tobramycin can suppress the

96 emergence of cheaters in cooperating PA14 populations grown on casein, similar to previous

97 observations with $C$. subtsugae (10). We sequenced the genomes of isolates from tobramycin-

98 evolved populations. All of the isolates had mutations in or upstream of $p t s P$, a gene coding for

99 phosphoenolpyruvate-protein phosphotransferase $\left(\mathrm{EI}^{\mathrm{Ntr}}\right)$. This enzyme is the first in a global

100 regulatory system known as the nitrogen phosphotransferase system $\left(\mathrm{PTS}^{\mathrm{Ntr}}\right)(27)$. Mutations in

101 pts $P$ were previously shown to increase LasR-I activity through a mechanism that is not well

102 understood (28). Interestingly, we observed that pts $P$ mutations suppress cheaters, even in 
103

104

105

106

107

108

109

110

111

112

113

114

115

116

117

118

119

120

121

122

123

124

125

populations passaged with no antibiotic. We demonstrate the cheater suppression is due to the QS-controlled toxin pyocyanin, which polices cheaters by selectively blocking their proliferation. Our results show that genetic adaptation to tobramycin can enhance policing against social cheaters. These results provide new information on policing mechanisms in $P$. aeruginosa and demonstrate how antibiotic selection can lead to changes in cooperative activity.

\section{MATERIALS AND METHODS}

\section{Culture conditions and reagents}

Bacteria were routinely grown in Luria-Bertani broth (LB) or LB buffered to $\mathrm{pH} 7$ with $50 \mathrm{mM}$ 3-(morpholino)-propanesulfonic acid (MOPS), or on LB agar (LBA; 1.5\% (weight per volume) Bacto-Agar). Growth media for specific experiments were M9-caseinate (casein broth; $6 \mathrm{~g} \mathrm{l}^{-1}$ $\mathrm{Na}_{2} \mathrm{HPO}_{4}, 3 \mathrm{~g} \mathrm{l}^{-1} \mathrm{KH}_{2} \mathrm{PO}_{4}, 0.5 \mathrm{~g} \mathrm{l}^{-1} \mathrm{NaCl}, 1 \mathrm{~g} \mathrm{l}^{-1} \mathrm{NH}_{4} \mathrm{Cl}$; $\mathrm{pH}$ 7.4, $1 \%$ sodium caseinate); MOPS minimal medium (25 mM D-glucose, freshly prepared $5 \mu \mathrm{M} \mathrm{FeSO}_{4}, 15 \mathrm{mM} \mathrm{NH} 4 \mathrm{Cl}$, and $2 \mathrm{mM}$ $\mathrm{K}_{2} \mathrm{HPO}_{4}$ added to a $1 \mathrm{X}$ MOPS base buffer consisting of $50 \mathrm{mM}$ MOPS, $4 \mathrm{mM}$ tricine, $50 \mathrm{mM}$ $\mathrm{NaCl}, 1 \mathrm{mM} \mathrm{K} \mathrm{SO}_{4}, 50 \mu \mathrm{M} \mathrm{MgCl}_{2}, 10 \mu \mathrm{M} \mathrm{CaCl}_{2}, 0.3 \mu \mathrm{M}\left(\mathrm{NH}_{4}\right)_{6} \mathrm{Mo}_{7} \mathrm{O}_{24}, 40 \mu \mathrm{M} \mathrm{H}_{3} \mathrm{BO}_{3}, 3 \mu \mathrm{M}$ $\mathrm{Co}(\mathrm{OAc})_{2}, 1 \mu \mathrm{M} \mathrm{CuSO}_{4}, 8 \mu \mathrm{M} \mathrm{MnSO}_{4}$, and $1 \mu \mathrm{M} \mathrm{ZnSO}_{4}$ ) (29); a modified M9-casamino acid broth (200 $\mathrm{ml} \mathrm{l}^{-1}$ 10X M9, $0.6 \mathrm{~g} \mathrm{l}^{-1}$ thiamine hydrochloride, $1 \mathrm{ml} \mathrm{l}^{-1} \quad 1 \mathrm{M} \mathrm{MgSO}_{4}, 1 \mathrm{ml} \mathrm{l}^{-1} \quad 0.2 \mathrm{M}$ $\mathrm{CaCl}_{2}, 10 \mathrm{~g} \mathrm{l}^{-1}$ casamino acid; freshly prepared; filter sterilized), Pyocyanin Producing Media (PPM) (30), and 4\% skim milk agar (SMA) (9). All P. aeruginosa broth cultures were grown at $37^{\circ} \mathrm{C}$ with shaking at $250 \mathrm{rpm}, 18 \mathrm{~mm}$ test tubes (for $2 \mathrm{ml}$ cultures), $125 \mathrm{ml}$ baffled flasks (10 ml cultures), or $250 \mathrm{ml}$ baffled flasks (60 ml cultures), unless otherwise specified. For E. coli, 100 $\mu \mathrm{g} \mathrm{ml} l^{-1}$ carbenicillin, $15 \mu \mathrm{g} \mathrm{ml}^{-1}$ gentamicin, and $10 \mu \mathrm{g} \mathrm{ml}^{-1}$ tetracycline were used. For $P$. aeruginosa, $300 \mu \mathrm{g} \mathrm{ml}^{-1}$ carbenicillin, 50-200 $\mathrm{g} \mathrm{ml}^{-1}$ gentamicin, and $200 \mu \mathrm{g} \mathrm{ml}^{-1}$ tetracycline 
126

127

128

129

130

131

132

133

134

135

136

137

138

139

140

141

142

143

144

145

146

147

were used. AHLs were purchased from Cayman Chemicals (MI, USA) and handled as described elsewhere (10). Genomic or plasmid DNA was extracted using Qiagen Puregene Core A kit (Hilden, Germany) or IBI Scientific plasmid purification mini-prep kit (IA, USA) while PCR products were purified using IBI Scientific PCR clean-up/gel extraction kits, according to the manufacturer's protocol. All antibiotics were purchased from GoldBio (MO, USA) except for tetracycline which is from Fisher Scientific (PA, USA). Pyocyanin and DMSO (solvent for pyocyanin) were purchased from Sigma Aldrich (MO, USA) and Acros Organics (WI, USA), respectively.

\section{Bacterial strains and strain construction}

All bacterial strains, plasmids, and primers used in this study are listed in Tables S1-S3. $P$. aeruginosa strain UCBPP-PA14 ('PA14') and PA14 derivatives were used for these studies. Markerless deletions in specific loci of $P$. aeruginosa PA14 were generated using allelic exchange as described previously (31). To generate plasmids for allelic exchange, DNA fragments with the mutated or deleted gene allele plus 500 bp flanking DNA were generated by PCR using primer-incorporated restriction enzyme sites. The PCR product was digested and ligated to pEXG2 (fusAl G1634A) or pEX18Ap ( $\Delta l a s R$ and $\Delta r h l R$ ) and transformed into the appropriate $P$. aeruginosa strain. The plasmids for $\Delta l a s I$ and $\Delta p t s P$ were described elsewhere $(27,32)$. Merodiploids were selected on Pseudomonas Isolation Agar (PIA)-carbenicillin (300 $\mu \mathrm{g}$

$\mathrm{ml}^{-1}$ ) for $\Delta l a s R$ and $\Delta r h l R$, PIA-gentamicin $\left(200 \mu \mathrm{g} \mathrm{ml}{ }^{-1}\right)$ for $\Delta l a s I$ and $\Delta p t s P$, and LBAgentamicin $\left(50 \mu \mathrm{g} \mathrm{ml}^{-1}\right)$ for fusAl G1634A. Deletion mutants were counterselected using $\mathrm{NaCl}$ free-15\% sucrose. Putative mutants were verified through antibiotic sensitivity tests and genetargeted Sanger sequencing. Plasmid transformations were described previously (27, 33-35). 
148 Complementation strains were constructed by integrating the mini $\square$ CTX $\square 1$ vector at the neutral

149 chromosomal attB locus $(27,34)$.

\section{Evolution experiments}

151 To prepare the inoculum for evolution experiments, overnight (18 h) pure cultures were grown in

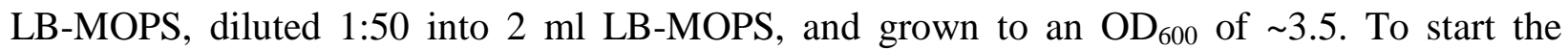
experiment, $50 \mu \mathrm{l}$ from this starter culture was transferred to $2 \mathrm{ml}$ fresh casein broth in an $18 \mathrm{~mm}$ tube. At $24 \mathrm{~h}$ intervals, cultures were diluted 1:50 into fresh casein broth in a new tube.

155 Tobramycin was added every other day where indicated, similar to previous experiments with $C$.

156 subtsugae (10). $\mathrm{CFU} \mathrm{m} \mathrm{m}^{-1}$ for each lineage was determined by viable plate counts every $96 \mathrm{~h}$. The $157 \%$ las $R$ mutant cheater (lasR cheater) was determined by patching 100 colonies, unless otherwise 158 specified, on SMA. Sixteen lasR mutants were sequence-verified by Sanger sequencing (Table 159 S4).

\section{Antimicrobial susceptibility assay}

161 Tobramycin susceptibility was determined by MIC according to the 2020 guidelines of the 162 Clinical and Laboratory Standards Institute (CSLI), using a modified dilution method. Briefly, 163 tobramycin was added to MOPS minimal medium and successively diluted 2-fold in a $200 \mu \mathrm{l}$ 164 volume in $2 \mathrm{ml}$ tubes. For each experiment, two dilution series were staggered by starting them 165 at different tobramycin concentrations to cover a broader range of concentrations. $P$. aeruginosa 166 inocula were from LB-grown stationary-phase cells $\left(\mathrm{OD}_{600}\right.$ of $\left.3.2-4\right)$, being careful to ensure that 167 all cultures in each experiment were grown to an identical cell density. The cultures were diluted 168 1:40 into each tube containing tobramycin, and the tubes were incubated with shaking for $20 \mathrm{~h}$. 169 After incubation, turbidity was measured using a BioTek Synergy 2 plate reader. The MIC was 
170 defined as the lowest concentration of tobramycin $\left(\mu \mathrm{g} \mathrm{ml}{ }^{-1}\right)$ in which bacterial growth was not

171 measurable. In some cases, $\mathrm{CFU} \mathrm{ml^{-1 }}$ was also determined by viable plate counts.

To determine susceptibility to $P$. aeruginosa culture fluid, we prepared culture fluid by inoculating overnight cultures to an optical density at $600 \mathrm{~nm}\left(\mathrm{OD}_{600}\right)$ of 0.1 into $60 \mathrm{ml}$ casein

174 broth, grew these cultures for $20 \mathrm{~h}$, then passed cultures through a $0.22 \mu \mathrm{m}$ filter to remove cells.

175 The filtered fluid was mixed with $100 \mu \mathrm{l}$ M9-casamino acid broth to a final volume of $2 \mathrm{ml}$ and

176 this was inoculated at an initial $\mathrm{OD}_{600}$ of 0.001 with either wild type, $\Delta$ lasR, $\Delta p t s P$, or $\Delta p t s P$

$177 \Delta$ lasR from logarithmic-stage LB-MOPS cultures at an $\mathrm{OD}_{600}$ 0.2-0.6. The M9-casamino-filtrate

178 mixture was incubated for $24 \mathrm{~h}$ and the initial and final population counts $\left(\mathrm{CFU} \mathrm{ml} \mathrm{l}^{-1}\right)$ were 179 enumerated by colony counting on plates.

180 Whole genome sequencing

181 Genomic DNA was extracted using the Qiagen Puregene yeast/bacteria kit and a sequencing 182 library was constructed with 350-bp inserts (strain T2) or 200-bp inserts (all other strains). 183 Sequencing was performed using Illumina HiSeq 4000 (for strain T2) or Illumina MiSeq with $184 \sim 25 \mathrm{X}$ coverage (all other strains). The raw reads were aligned to the $P$. aeruginosa UCBPP185 PA14 reference genome (UCBPP-PA14 Accession NC_008463) using Strand NGS (Bangalore, 186 India) software v 3.1.1, using a pipeline described previously (36). Mutations of interest were 187 verified by gene-targeted Sanger sequencing. Sequence reads for the ancestral PA14 188 (SAMN16823471) and tobramycin-evolved isolates (SAMN16823472 - SAMN16823478) can 189 be found at the NCBI SRA under BioProject PRJNA678537.

190 Measurements of LasR activity and pyocyanin production. To measure LasR activity, we 191 first introduced the LasR-responsive plasmid pBS351 to $P$. aeruginosa mutants or wild-type 192 PA14 strains by electroporation (35). Electrocompetent cells were prepared from overnight 
193 cultures using $300 \mathrm{mM}$ sucrose (37). Transformants were selected on LB agar using gentamicin 194 at 50-200 $\mathrm{g} \mathrm{ml}^{-1}$ and routinely grown with gentamicin $\left(50 \mu \mathrm{g} \mathrm{ml}^{-1}\right.$ for agar and $15 \mu \mathrm{g} \mathrm{ml}^{-1}$ for 195 broth) for plasmid maintenance. Transformants were grown in LB-MOPS with $15 \mu \mathrm{g} \mathrm{m}{ }^{-1}$ 196 gentamicin for $18 \mathrm{~h}$, washed with phosphate buffered saline (PBS), and fluorescence was 197 measured using a BioTek Synergy 2 plate reader. For pyocyanin measurements, we extracted 198 pyocyanin as described previously (38-40). Briefly, cells were grown for $18 \mathrm{~h}$ in Pyocyanin199 producing media (30), and $5 \mathrm{ml}$ whole culture was extracted with $2 \mathrm{ml}$ chloroform. The organic 200 layer was separated and extracted a second time with $0.2 \mathrm{~N} \mathrm{HCl}$. The absorbance of the aqueous 201 layer was measured at $520 \mathrm{~nm}$ and multiplied by 17.072 to calculate the pyocyanin concentration

$202\left(\mu \mathrm{g} \mathrm{ml}^{-1}\right)(39)$. LasR activity and pyocyanin measurements were normalized to culture density 203 (optical density at $600 \mathrm{~nm}$ ) for reporting data.

204 Coculture assays

205 Coculture experiments were conducted in $2 \mathrm{ml}$ casein broth in $18 \mathrm{~mm}$ test tubes. To prepare the 206 inoculum, overnight (18 h) pure cultures were grown in LB-MOPS, diluted to an optical density 207 at $600 \mathrm{~nm}\left(\mathrm{OD}_{600}\right)$ of 0.025 for cheaters or 0.05 for cooperators into LB-MOPS, and grown to an $208 \mathrm{OD}_{600}$ of $\sim 3.5$ before combining at a 99:1 (cooperator:cheater) ratio. This mixture was then 209 diluted 1:40 into casein broth to start the coculture. Cocultures were diluted 1:40 into fresh 210 casein broth in a new tube every $24 \mathrm{~h}$ until the end of the experiment at $72 \mathrm{~h}$. The initial and final 211 total population counts $\left(\mathrm{CFU} \mathrm{ml}{ }^{-1}\right)$ were determined by viable plate counts. The $\%$ lasR cheater 212 was determined by patching 200 colonies on SMA. 
As an initial test that LasR contributes to tobramycin resistance in planktonic conditions,

217 we determined the minimum inhibitory concentration (MIC) of tobramycin against the

218 laboratory strain PA14 or PA14 with a deletion of lasR or lasI. To increase the MIC detection

219 sensitivity, we generated two tobramycin dilution series that were staggered by using two

220 different starting concentrations (41). We observed a small but reproducible 1.7-fold decrease in

221 the $\Delta l a s R$ mutant MIC relative to PA14 (Fig. 1A). The difference was observed when

222 tobramycin was used to treat cultures grown to an optical density at $600 \mathrm{~nm}$ of $4\left(\mathrm{OD}_{600} 4\right)$, but

223 not cultures at lower cell densities (Fig. S1). After $24 \mathrm{~h}$ treatment with $1.1 \mu \mathrm{g} \mathrm{ml} \mathrm{l}^{-1}$ tobramycin,

224 about 200-fold fewer $\Delta l a s R$ mutant cells were recovered than wild type (Fig. 1B). We also

225 observed a decrease in the MIC of the $\Delta l a s I$ mutant similar to that of the $\Delta l a s R$ mutant (Fig. 1A).

226 We could restore resistance to the $\Delta$ lasI mutant by adding synthetic 3OC12-HSL (Fig. 1A).

227 There was no significant difference between the wild type and the $\Delta r h l R$ mutant, supporting the

228 hypothesis that the RhlR system is not important for the resistance phenotype. Further, the MIC

229 of the $\Delta l a s R, \Delta r h l R$ double mutant was similar to that of the $\Delta l a s R$ single mutant. Together,

230 these results show that the LasR-I system, but not the RhIR-I system, contributes to tobramycin

231 resistance in planktonically-grown $P$. aeruginosa strain PA14.

\section{Tobramycin suppresses cheating in $P$. aeruginosa}

P. aeruginosa requires a QS-controlled protease to grow in minimal medium with casein

234 as the sole source of carbon and energy, which can be exploited by lasR-mutated cheaters that

235 emerge in populations repeatedly passaged under these conditions $(8,9)$. We hypothesized that

236 tobramycin might suppress lasR mutants in cooperating $P$. aeruginosa populations passaged on

237 casein. To test this hypothesis, we assessed the frequency of lasR mutants in $P$. aeruginosa

238 populations in casein broth or in broth with $0.6 \mu \mathrm{g} \mathrm{ml}{ }^{-1}$ tobramycin, a concentration determined 
239

240

241

242

243

244

245

246

247

248

249

250

251

252

253

254

255

256

257

258

259

260

261

to be sub-MIC in these conditions. We distinguished lasR mutants by colony phenotypes (42, 43), which was confirmed in a subset of mutants by sequencing the lasR gene (Table S3). In populations with no antibiotic, lasR mutants emerged between 5-8 days and increased to $>99 \%$ of the population (Fig. 2). None of our populations showed evidence of a collapse, as has been reported in similar experiments with PAO1 $(9,13,16)$. In the populations with tobramycin, lasR mutant frequencies remained low or below the detection level throughout the experiment (Fig. 2). These results show that tobramycin can suppress the emergence of LasR-mutated cheaters in casein-grown $P$. aeruginosa populations.

\section{Variants from tobramycin-treated populations undergo genetic adaptation}

To assess how antibiotic adaptation influences cheater emergence and suppression, we carried out a second experiment using higher tobramycin concentrations for stronger selection. We used an initial concentration of $0.6 \mu \mathrm{g} \mathrm{m} \mathrm{m}^{-1}$ tobramycin and increased the concentration by $50 \%$ every 4 days to reach a final concentration of $7.1 \mu \mathrm{g} \mathrm{ml}^{-1}$. In three independent cultures, the lasR mutant population remained at less than $5 \%$ of the total population (Fig. 2). We did not observe any significant growth inhibition at any stage of the experiment, even at the highest tobramycin concentration (Fig. S2), suggesting genetic adaptation occurred during passage. To test this hypothesis, we determined the MIC of one isolated variant from each of the passaged populations (variants $\mathrm{T} 1, \mathrm{~T} 2$, and $\mathrm{T} 3$ from populations passaged with $0.6-7.1 \mu \mathrm{g} \mathrm{ml} \mathrm{l}^{-1}$ tobramycin and variants $\mathrm{T} 4, \mathrm{~T} 5$, and T6 from populations passaged with $0.6 \mu \mathrm{g} \mathrm{ml} \mathrm{m}^{-1}$ tobramycin). All six variants showed a higher tobramycin MIC than the ancestor strain (Fig. S3A) or variants from identically treated populations with no added antibiotic (clones N1-N3, Fig. S3B).

To identify the mutations that accumulated in tobramycin-evolved variants, we sequenced the genomes of our six tobramycin-evolved variants. For comparison, we also 
262

263

264

265

266

267

268

269

270

271

272

273

274

275

276

277

278

279

280

281

282

283

284

sequenced the parent PA14 strain and clone N2 described above, an isolate from a population passaged with no antibiotic. We identified 3-6 mutations in each of the tobramycin-evolved variants that were not in either the parent PA14 or isolate N2 (Table 1 and S5). Most of the tobramycin-evolved variants had mutations in two genes: $p t s P$, which codes for phosphoenolpyruvate protein phosphotransferase and fusAl, which codes for translation elongation factor EF-G1A and is considered essential (44). To verify the role of ptsP and fusAl mutations in tobramycin resistance, we introduced mutations of each to the PA14 genome. We used $\triangle p t s P$ or the fusAl G1643A mutation from isolate T5, because fusAl deletions are thought to be nonviable (44). We also constructed a $\Delta p t s P$, fusAl G1634A double mutant. We compared the MIC of the mutated strains with that of the PA14 parent (Fig. S3C and S3D). Each of the individual mutations increased PA14 resistance by about 2-fold and combining mutations increased the MIC by about 3-fold (Fig S3E).

\section{pts $P$-mutated strains have enhanced LasR activity and pyocyanin production.}

We focused our attention on the pts $P$ mutation because disrupting $p t s P$ was previously shown to increase LasR activity (28), and we were interested in understanding how these effects could alter the cooperator-cheating dynamic. First, we tested the hypothesis that the LasR-LasI system is elevated at least in the T1, T2 and T3 mutants. To do so, we used a PlasI-gfp plasmid reporter (35), in which the $g f p$ reporter gene is fused to the LasR-responsive lasI promoter. This reporter showed $\sim 2-3$-fold higher fluorescence in the $\triangle p t s P$ mutant than that of the wild-type PA14 strain (Fig. S4A), similar to previous results (28). The T1, T2 and T3 variants carrying this reporter also showed $\sim 3$-fold higher fluorescence than that of PA14. The elevated fluorescence levels in these variants could be restored to wild-type levels by introducing pts $P$ to the neutral 
285 286

287 288

289

290

291

292

293

294

295

296

297

298

299

300

301

302

303

304

305

306

307

$a t t B$ site in the genome (Fig. 3A). The reporter activities of isolates N1, N2, and N3 were similar to that of PA14 (Fig. S4B). The difference in reporter activity in the PA14 $\Delta p t s P$ mutant or the T1-T3 strains could not be explained by differences in growth (Table S6).

Disruptions in $p t s P$ were previously shown to increase pyocyanin production by about 3fold (28). To test whether pyocyanin production was similarly elevated in our tobramycinevolved variants, we compared pyocyanin produced by the T1, T2, and T3 variants with that of PA14. Our results showed the variants have $\sim 3$-fold higher pyocyanin production than the PA14 parent, and that pyocyanin production can be reduced to wild-type levels in the variants by introducing pts $P$ to the $a t t B$ site in the genome (Fig. 3B). This increase was not observed in isolates N1-N3 (Fig. S4C). Thus, ptsP mutations also increase pyocyanin production in strains passaged with tobramycin.

\section{lasR-mutant cheaters are suppressed in $p t s P$ mutant populations.}

Because pts $P$ mutations alter QS regulation, we predicted that these mutations might influence cheating dynamics even in the absence of tobramycin. Specifically, we predicted las $R$ mutant cheaters might emerge at a faster rate in $p t s P$ mutant populations due to the metabolic burden associated with the higher LasR activity. To test this hypothesis, we passaged the T1, T2, and T3 variants in casein broth for 32 days in the absence of antibiotic and monitored the emergence of lasR-mutant cheaters (Fig. 4A and S5). Surprisingly, cheaters emerged later in these populations compared with that of the PA14 ancestor (23 to >32 days for T1-T3 vs. 6-8 days for PA14). For T2, cheaters were never observed even by 32 days. Further, the total cheater

frequency in the T1 and T3 populations did not reach the same levels as that of PA14 (33-67\% for T1 and T3 vs. >98\% for PA14) (Fig. S5A). There were no differences in cheating observed 
with variants N1-N3 passaged with no tobramycin, compared with PA14 (Fig. S5C). The total cell density remained similar for all evolution experiments, suggesting the differences in cheating were not due to differences in total population of the experiments (Fig. S5B, S5D).

We hypothesized that the difference in cheating observed with the tobramycin-evolved variants was due to the disrupted $p t s P$ gene in these strains. To test this hypothesis, we tested cheating in a $\Delta p t s P$ mutant. We passaged the $\Delta p t s P$ mutant carrying either CTX-1 or the CTX-1pts $P$ cassette in $1 \%$ casein broth for 32 days and monitored cheater frequency over time. We also monitored cheating in PA14 and PA14 CTX-1. We observed no differences in cheating of PA14 CTX-1 compared with PA14, verifying that the CTX-1 cassette had no effect on cheating. However, cheaters emerged later and reached lower levels overall in the $\Delta p t s P-\mathrm{CTX}-1$ populations compared with either PA14 strain (Fig. 4B, S5E). Cheating was indistinguishable from PA14 in the $\Delta p t s P$-CTX-1-ptsP populations (Fig. 4B, S5E). There were no notable differences in total growth of any of these populations (Fig. S5F). Together, our results show that pts $P$ mutations cause delayed cheating and reduced cheater loads.

\section{Pyocyanin produced by $\Delta p t s P$ mutants is active against las $R$-mutated cheaters}

A potential explanation for delayed cheating in the $\Delta p t s P$ mutant populations is that the $\Delta p t s P$ mutant has a growth advantage compared with the PA14 ancestral strain. However, PA14 and $\Delta p t s P$ showed identical growth rates (Table S6), suggesting there is another explanation. We hypothesized that the $\Delta p t s P$ mutant produces a toxin that inhibits growth of las $R$ mutant cheaters. To test this hypothesis, we filtered culture fluid from a $\Delta p t s P$ mutant and tested its ability to inhibit growth of logarithmically growing PA14 or PA14 with deletions of ptsP, lasR, or both $p t s P$ and lasR. We performed identical treatments with filtered fluid from PA14 or with 
331

332

333

334

335

336

unspent culture media (untreated control) and enumerated cell growth after $24 \mathrm{~h}$. Treatment with PA14 culture fluid had no effects on growth and was similar to the untreated control (Fig. 5). However, treatment with the $\Delta p t s P$ mutant filtrate reduced growth of the $\Delta l a s R$ mutant by 83 fold and $\Delta p t s P \Delta l a s R$ mutants by 13,000 -fold, compared with no treatment. Thus, the $\Delta p t s P$ mutant secretes a substance that is growth inhibitive to las $R$-mutated strains and particularly to $\triangle p t s P \Delta l a s R$ mutants.

We hypothesized that the activity in the $\Delta p t s P$ mutant culture fluid was due to pyocyanin, based on our result that ptsP mutations increase pyocyanin production (Fig. 3). Pyocyanin is produced from the phzA1-G1 (phzl) and phzA2-G2 (phz2) gene clusters and the $p h z M$ and $p h z S$ genes (Fig. 6A) (45, 46). Several of the pyocyanin intermediates are toxic, such as 5methylphenazine 1-carboxylic acid (5-Me-PCA) (47-49). We tested several gene mutations in this pathway to determine whether any of the products have activity against lasR mutants. We deleted $p h z M$, which is needed to convert the precursor phenazine-1-carboxylic acid (PCA) to 5Me-PCA, $p h z S$ which is needed to convert 5-Me-PCA to pyocyanin $(45,50)$, and $p h z H$ which is not needed for pyocyanin production but involved in production of another product of this pathway, phenazine-1-carboxamide (PCN) (45).

We initially tested whether pyocyanin biosynthesis contributes to the antimicrobial activity of a ptsP mutant by making a $\Delta p t s P \Delta p h z 1 \quad \Delta p h z 2$ triple mutant. We compared antimicrobial activity in culture fluid of this strain with that of the single $\Delta p t s P$ mutant, and tested against the $\Delta p t s P, \Delta l a s R$ mutant. Deleting both $p h z 1$ and $p h z 2$ abolished the antimicrobial activity observed with the $\Delta p t s P$ single mutant (Fig. 6B). Mutations in $p h z M$ or $p h z S$ similarly abolished this activity (Fig. 6B). However, no effects were observed by deleting $p h z H$ (Fig. 6B), which is important for production of the pyocyanin bi-product PCN but not pyocyanin itself. 
354 These results support that the antimicrobial activity in $\Delta p t s P$ mutant culture fluid is due to

355 pyocyanin. We also tested whether commercially supplied pyocyanin could restore antimicrobial

356 activity of the $\Delta p t s P \Delta p h z S$ mutant by adding $10 \mu \mathrm{g} \mathrm{ml}^{-1}$ pyocyanin to $\Delta p t s P \Delta p h z S$ culture

357 filtrates prior to treating $\triangle p t s P \Delta l a s R$ cells. Adding pyocyanin reduced $\Delta p t s P, \Delta l a s R$ mutant

358 growth $\sim 10^{7}$-fold, whereas PA14 growth was only reduced by $\sim 10$-fold (Fig. 6C). Together,

359 these results support the conclusion that pyocyanin can selectively limit growth of $\Delta p t s P, \Delta l a s R$ 360 mutants.

\section{Pyocyanin production leads to enhanced policing by $\Delta p t s P$ mutants.}

We also tested the role of pyocyanin in policing cheaters using competition experiments.

We mixed the $\Delta l a s R$ mutant with a cooperator starting at a 1:99 (cheater:cooperator) ratio in $1 \%$

\section{DISCUSSION}

This study adds to the evidence that quorum sensing contributes to antibiotic resistance in

376 Proteobacteria. Results from these studies show that $P$. aeruginosa LasR increases tobramycin 
377 resistance in planktonic PA14 cultures. The results also show that tobramycin can limit the

378 proliferation of lasR mutant cheaters in cooperating populations grown on casein. Cheaters are

379 suppressed directly by tobramycin because they are more susceptible to growth-inhibiting

380 effects. Cheaters are also suppressed, indirectly, by tobramycin-selected adaptive mutations that

381 led to pyocyanin-dependent policing. Together, these results support the idea that antibiotics

382 influence quorum sensing stability directly and indirectly through adaptive changes in the 383 population.

These results also provide an example of how adaptive mutations can alter cheater control mechanisms. Adaptive mutations have been shown to influence cooperation and cheating in other studies (51-53). For example, adaptive mutations can improve the ability of cooperators to outcompete cheaters $(19,52)$ or cause re-wiring of cooperative traits and allow the cheaters to cooperate again (51). In this study, once adaptive mutations emerge in the population they caused suppression of cheaters through policing. Thus, antibiotic exposure stabilized cooperation through heritable changes that led to policing. These antibiotic-selected effects on cheating could

391 have important implications for understanding the long-term consequences of antibiotic usage 392 for treating infections.

394 pyocyanin. Pyocyanin was previously shown to be involved in policing las $R$ mutant cheaters (17, 395 54). Pyocyanin leads to the generation of highly toxic hydroxyl radicals (55-57), which can cause 396 oxidative stress and cell death $(58,59)$. LasR mutant cheaters fail to upregulate enzymes 397 involved in relieving oxidative stress such as catalase and superoxide dismutase (SOD) (17, 56, $39858,60)$, which explains why these mutants might be more sensitive to pyocyanin than the wild 399 type $(60,61)$. However, these detoxifying enzymes can benefit both producing and non- 
400

401

402

403

404

405

406

407

408

409

410

411

412

413

414

415

416

417

418

419

420

421

422

producing members of the population (19), which may explain how lasR cheaters were still able to increase in frequency when cocultured with the cooperator, despite the policing effect of pyocyanin (Fig. 6). It will be interesting to determine whether pyocyanin production could have similar effects on stabilizing quorum sensing in natural communities, such as infections.

Pyocyanin production was increased due to mutations in $p t s P$. PtsP, along with two other enzymes PtsN and PtsO, make up a poorly understood system called the nitrogen phosphotransferase system $\left(\mathrm{PTS}^{\mathrm{Ntr}}\right)$. In Escherichia coli, $\mathrm{PTS}^{\mathrm{Ntr}}$ is involved in regulating diverse physiological changes in response to nitrogen starvation $(62,63)$. It is unclear if PTS ${ }^{\mathrm{Ntr}}$ plays a similar role in $P$. aeruginosa. In $P$. aeruginosa, PtsP contributes to pathogenesis (64), biofilm formation (27), and quorum sensing regulation (28). Mutations in $p t s P$ have also been shown to contribute to tobramycin resistance $(65,66)$, consistent with our results. The specific effects of PtsP on pathogenesis, biofilm formation, and tobramycin resistance are as-yet unknown. In the case of quorum sensing, it is thought that PtsP somehow represses LasR through the antiactivator QscR (28), although it is not clear if PtsP acts entirely through QscR or modifies quorum sensing through other pathways. The important effects of mutating PtsP on P. aeruginosa virulence and virulence-associated behaviors suggest PtsP and the $\mathrm{PTS}^{\mathrm{Ntr}}$ system might have potential as a new target for therapeutic development.

Our results also show that quorum sensing has a small but appreciable contribution to antibiotic resistance in $P$. aeruginosa strain PA14 under planktonic conditions (Fig. 1). Similar results have been reported for other strains and species, for example in $P$. aeruginosa PAO1 in biofilms (20-23), and C. subtsugae (10). In C. subtsugae, resistance is attributed to an efflux pump, CdeAB-OprA, which is transcriptionally activated by the CviR quorum-sensing receptor in response to the cognate quorum-sensing signal $N$-hexanoyl-homoserine lactone $(10,41)$. In $P$. 
423 aeruginosa, there may be multiple factors contributing to antibiotic resistance. There are at least

424 three efflux pumps known to have overlapping specificity for aminoglycosides; MexAB $(67,68)$,

425 MexXY (69, 70), and PA1874-1877 (71); there are also aminoglycoside-inactivating enzymes

426 (67). Quorum-control of antibiotic resistance may provide an important evolutionary benefit. For

427 example, quorum sensing may increase resistance to protect against self-produced toxins, or

428 synchronize resistance factor expression across members of the population (72) to protect

429 neighboring cells from exported antibiotic (73). Understanding how and why quorum sensing

430 contributes to antibiotic resistance will provide important new information about the biology of

431 quorum sensing and will be relevant to designing new therapies that function by blocking

432 quorum-sensing systems.

433

434 ACKNOWLEDGEMENTS

435 This work was supported by the NIH through grant R35GM133572 to JRC and grant GM125714

436 to AAD, a pilot project from the Chemical Biology of Infectious Diseases (P20 GM113117)

437 program to JRC, and a KU Inez Jay award to JRC. Sequencing core facility support was

438 provided by the NIH COBRE Center for Molecular Analysis of Disease Pathways Program (P20

439 GM103638). JHK was supported by an NIH postdoctoral fellowship (T32 AI007343). VDC was

440 supported by an Undergraduate Research Award from the KU Center for Undergraduate

441 Research and a K-INBRE fellowship (P20 GM103418). RGA was supported by the Fulbright

442 Foreign Student Program (15160174). The authors would also like to acknowledge Matthew

443 Parsek and Dingding An (University of Washington), Matthew Cabeen (Oklahoma State

444 University) and Lars Dietrich (Columbia University) for providing P. aeruginosa strains and

445 plasmids, as well as Tony Ma, Matthew Johnson, and Bryan Murphy for their technical support. 


\section{AUTHOR CONTRIBUTIONS}

448 R.G.A. contributed to the conception and design of the work, the acquisition, analysis and 449 interpretation of data, and drafting and revising the manuscript. J.H.K. contributed to the design 450 of the work, the acquisition, analysis and interpretation of data and revising the manuscript. 451 B.M.M. contributed to the acquisition and analysis of data. V.D.C. contributed to the acquisition 452 and analysis of data. N.E.S. contributed to the analysis and interpretation of data. A.A.D. 453 contributed to the analysis and interpretation of data. J.R.C. contributed to the design of the 454 work, analysis and interpretation of data, and drafting and revising the manuscript.

\section{CONFLICT OF INTEREST}

458 The authors declare that they have no conflict of interest.

\section{REFERENCES}

460 1. Fuqua WC, Winans SC, Greenberg EP. Quorum sensing in bacteria: the LuxR-LuxI 461 family of cell density-responsive transcriptional regulators. Journal of Bacteriology. $462 \quad 1994 ; 176(2): 269-75$.

463 2. Papenfort K, Bassler BL. Quorum sensing signal-response systems in gram-negative 464 bacteria. Nature Reviews Microbiology. 2016;14(9):576-88.

465 3. Schuster M, Sexton DJ, Diggle SP, Greenberg EP. Acyl-homoserine lactone quorum 466 sensing: From evolution to application. Annual Review of Microbiology. 2013;67(1):43-63.

467 4. Fuqua C, Greenberg EP. Listening in on bacteria: acyl-homoserine lactone signalling. 468 Nature Reviews Molecular Cell Biology. 2002;3(9):685-95. 
5. Fuqua C, Winans SC, Greenberg EP. Census and consensus in bacterial ecosystems: The LuxR-LuxI family of quorum-sensing transcriptional regulators. Annual Review of Microbiology. 1996;50(1):727-51.

6. Whiteley M, Diggle SP, Greenberg EP. Progress in and promise of bacterial quorum sensing research. Nature. 2017;551:313-20.

7. West SA, Griffin AS, Gardner A, Diggle SP. Social evolution theory for microorganisms. Nature Reviews Microbiology. 2006;4:597.

8. Diggle SP, Griffin AS, Campbell GS, West SA. Cooperation and conflict in quorumsensing bacterial populations. Nature. 2007;450:411.

9. Sandoz KM, Mitzimberg SM, Schuster M. Social cheating in Pseudomonas aeruginosa quorum sensing. Proceedings of the National Academy of Sciences. 2007;104(40):15876-81.

10. Evans KC, Benomar S, Camuy-Vélez LA, Nasseri EB, Wang X, Neuenswander B, et al. Quorum-sensing control of antibiotic resistance stabilizes cooperation in Chromobacterium violaceum. The ISME Journal. 2018;12(5):1263-72.

11. West SA, Diggle SP, Buckling A, Gardner A, Griffin AS. The social lives of microbes. Annual Review of Ecology, Evolution, and Systematics. 2007;38(1):53-77.

12. Hardin G. The tragedy of the commons. Science. 1968;162(3859):1243.

13. Dandekar AA, Chugani S, Greenberg EP. Bacterial quorum sensing and metabolic incentives to cooperate. Science 2012;338(6104):264-6.

14. Rainey PB, Rainey K. Evolution of cooperation and conflict in experimental bacterial populations. Nature. 2003;425(6953):72-4.

15. Clutton-Brock TH, Parker GA. Punishment in animal societies. Nature. 1995;373(6511):209-16.

16. Wang M, Schaefer AL, Dandekar AA, Greenberg EP. Quorum sensing and policing of Pseudomonas aeruginosa social cheaters. Proceedings of the National Academy of Sciences of the United States of America. 2015;112(7):2187-91. 
17. Castañeda-Tamez P, Ramírez-Peris J, Pérez-Velázquez J, Kuttler C, Jalalimanesh A, Saucedo-Mora MÁ, et al. Pyocyanin restricts social cheating in Pseudomonas aeruginosa. Frontiers in Microbiology. 2018;9:1348-.

18. Yan H, Asfahl KL, Li N, Sun F, Xiao J, Shen D, et al. Conditional quorum-sensing induction of a cyanide-insensitive terminal oxidase stabilizes cooperating populations of Pseudomonas aeruginosa. Nat Commun. 2019;10(1):4999.

19. Schuster M, Sexton DJ, Hense BA. Why quorum sensing controls private goods. Frontiers in Microbiology. 2017;8(885).

20. Bjarnsholt T, Jensen PO, Burmolle M, Hentzer M, Haagensen JA, Hougen HP, et al. Pseudomonas aeruginosa tolerance to tobramycin, hydrogen peroxide and polymorphonuclear leukocytes is quorum-sensing dependent. Microbiology. 2005;151(Pt 2):373-83.

21. Popat R, Crusz SA, Messina M, Williams P, West SA, Diggle SP. Quorum-sensing and cheating in bacterial biofilms. Proceedings of the Royal Society B: Biological Sciences. 2012;279(1748):4765-71.

22. Rasmussen TB, Skindersoe ME, Bjarnsholt T, Phipps RK, Christensen KB, Jensen PO, et al. Identity and effects of quorum-sensing inhibitors produced by Penicillium species. Microbiology. 2005;151:1325-40.

23. Shih P-C, Huang C-T. Effects of quorum-sensing deficiency on Pseudomonas aeruginosa biofilm formation and antibiotic resistance. Journal of Antimicrobial Chemotherapy. 2002;49(2):309-14.

24. Pearson JP, Passador L, Iglewski BH, Greenberg EP. A second N-acylhomoserine lactone signal produced by Pseudomonas aeruginosa. Proceedings of the National Academy of Sciences of the United States of America. 1995;92(5):1490-4.

25. Pesci EC, Pearson JP, Seed PC, Iglewski BH. Regulation of las and rhl quorum sensing in Pseudomonas aeruginosa. Journal of Bacteriology. 1997;179(10):3127-32.

26. Lee J, Zhang L. The hierarchy quorum sensing network in Pseudomonas aeruginosa. Protein \& Cell. 2015;6(1):26-41. 
522 27. Cabeen MT, Leiman SA, Losick R. Colony-morphology screening uncovers a role for the 523 Pseudomonas aeruginosa nitrogen-related phosphotransferase system in biofilm formation. 524 Molecular Microbiology. 2016;99(3):557-70.

525

526

527

528

529 530

28. $\mathrm{Xu} \mathrm{H}$, Lin $\mathrm{W}, \mathrm{Xia} \mathrm{H}, \mathrm{Xu} \mathrm{S}, \mathrm{Li} \mathrm{Y}$, Yao H, et al. Influence of ptsP gene on pyocyanin production in Pseudomonas aeruginosa. FEMS Microbiol Lett. 2005;253(1):103-9.

29. Welsh MA, Blackwell HE. Chemical genetics reveals environment-specific roles for quorum sensing circuits in Pseudomonas aeruginosa. Cell Chem Biol. 2016;23(3):361-9.

30. Wentworth BB. Diagnostic procedures for bacterial infections. Washington, DC: American Public Health Association; 1987.

31. Hmelo LR, Borlee BR, Almblad H, Love ME, Randall TE, Tseng BS, et al. Precisionengineering the Pseudomonas aeruginosa genome with two-step allelic exchange. Nature Protocols. 2015;10:1820-41.

32. Chugani S, Kim BS, Phattarasukol S, Brittnacher MJ, Choi SH, Harwood CS, et al. Strain-dependent diversity in the Pseudomonas aeruginosa quorum-sensing regulon. Proc Natl Acad Sci U S A. 2012;109(41):E2823-E31.

33. Choi K-H, Schweizer HP. mini-Tn7 insertion in bacteria with single attTn7 sites: example Pseudomonas aeruginosa. Nature Protocols. 2006;1(1):153-61.

34. Hoang TT, Kutchma AJ, Becher A, Schweizer HP. Integration-proficient plasmids for Pseudomonas aeruginosa: Site-specific integration and use for engineering of reporter and expression strains. Plasmid. 2000;43(1):59-72.

35. Feltner JB, Wolter DJ, Pope CE, Groleau M-C, Smalley NE, Greenberg EP, et al. LasR variant cystic fibrosis isolates reveal an adaptable quorum-sensing hierarchy in Pseudomonas aeruginosa. mBio. 2016;7(5):1-9.

36. Toussaint JP, Farrell-Sherman A, Feldman TP, Smalley NE, Schaefer AL, Greenberg EP, et al. Gene duplication in Pseudomonas aeruginosa improves growth on adenosine. Journal of bacteriology. 2017.

37. Choi KH, Kumar A, Schweizer HP. A 10-min method for preparation of highly electrocompetent Pseudomonas aeruginosa cells: application for DNA fragment transfer 
between chromosomes and plasmid transformation. Journal of microbiological methods. 2006;64(3):391-7.

38. Kurachi M. Studies on the Biosynthesis of Pyocyanine. (II) : Isolation and Determination of Pyocyanine. Bulletin of the Institute for Chemical Research, Kyoto University. 1958;36:17487.

39. Essar DW, Eberly L, Hadero A, Crawford IP. Identification and characterization of genes for a second anthranilate synthase in Pseudomonas aeruginosa: interchangeability of the two anthranilate synthases and evolutionary implications. Journal of Bacteriology. 1990;172(2):884900 .

40. Ding F, Oinuma K-I, Smalley NE, Schaefer AL, Hamwy O, Greenberg EP, et al. The Pseudomonas aeruginosa orphan quorum sensing signal receptor QscR regulates global quorum sensing gene expression by activating a single linked operon. mBio. 2018;9(4):e01274-18.

41. Benomar S, Evans KC, Unckless RL, Chandler JR. Efflux pumps in Chromobacterium species increase antibiotic resistance and promote survival in a co-culture competition model. Applied and environmental microbiology. 2019;85(19):e00908-19.

42. Hoffman LR, Kulasekara HD, Emerson J, Houston LS, Burns JL, Ramsey BW, et al. Pseudomonas aeruginosa lasR mutants are associated with cystic fibrosis lung disease progression. Journal of Cystic Fibrosis. 2009;8(1):66-70.

43. D'Argenio DA, Wu M, Hoffman LR, Kulasekara HD, Déziel E, Smith EE, et al. Growth phenotypes of Pseudomonas aeruginosa lasR mutants adapted to the airways of cystic fibrosis patients. Molecular Microbiology. 2007;64(2):512-33.

44. Bolard A, Plesiat P, Jeannot K. Mutations in gene fusAl as a novel mechanism of aminoglycoside resistance in clinical strains of Pseudomonas aeruginosa. Antimicrobial agents and chemotherapy. 2018;62(2):1-10.

45. Mavrodi DV, Bonsall RF, Delaney SM, Soule MJ, Phillips G, Thomashow LS. Functional analysis of genes for biosynthesis of pyocyanin and phenazine-1-carboxamide from Pseudomonas aeruginosa PAO1. Journal of Bacteriology. 2001;183(21):6454.

46. Recinos DA, Sekedat MD, Hernandez A, Cohen TS, Sakhtah H, Prince AS, et al. Redundant phenazine operons in Pseudomonas aeruginosa exhibit environment-dependent expression and differential roles in pathogenicity. Proceedings of the National Academy of Sciences of the United States of America. 2012;109(47):19420-5. 
47. Sakhtah H, Koyama L, Zhang Y, Morales DK, Fields BL, Price-Whelan A, et al. The Pseudomonas aeruginosa efflux pump MexGHI-OpmD transports a natural phenazine that controls gene expression and biofilm development. Proceedings of the National Academy of Sciences of the United States of America. 2016;113(25):E3538-47.

48. Cezairliyan B, Vinayavekhin N, Grenfell-Lee D, Yuen GJ, Saghatelian A, Ausubel FM. Identification of Pseudomonas aeruginosa phenazines that kill Caenorhabditis elegans. PLOS Pathogens. 2013;9(1):e1003101.

49. Gibson J, Sood A, Hogan DA. Pseudomonas aeruginosa-Candida albicans interactions: localization and fungal toxicity of a phenazine derivative. Applied and environmental microbiology. 2009;75(2):504-13.

50. Parsons JF, Greenhagen BT, Shi K, Calabrese K, Robinson H, Ladner JE. Structural and functional analysis of the pyocyanin biosynthetic protein PhzM from Pseudomonas aeruginosa. Biochemistry. 2007;46(7):1821-8.

51. Oshri RD, Zrihen KS, Shner I, Omer Bendori S, Eldar A. Selection for increased quorum-sensing cooperation in Pseudomonas aeruginosa through the shut-down of a drug resistance pump. The ISME journal. 2018;12(10):2458-69.

52. Waite AJ, Shou W. Adaptation to a new environment allows cooperators to purge cheaters stochastically. Proceedings of the National Academy of Sciences. 2012;109(47):1907986.

53. Asfahl KL, Walsh J, Gilbert K, Schuster M. Non-social adaptation defers a tragedy of the commons in Pseudomonas aeruginosa quorum sensing. The ISME Journal. 2015;9(8):1734-46.

54. Lai B-m, Yan H-c, Wang M-z, Li N, Shen D-s. A common evolutionary pathway for maintaining quorum sensing in Pseudomonas aeruginosa. Journal of Microbiology. 2018;56(2):83-9.

55. Rada B, Leto TL. Pyocyanin effects on respiratory epithelium: relevance in Pseudomonas aeruginosa airway infections. Trends in Microbiology. 2013;21(2):73-81.

56. Hassett DJ, Charniga L, Bean K, Ohman DE, Cohen MS. Response of Pseudomonas aeruginosa to pyocyanin: mechanisms of resistance, antioxidant defenses, and demonstration of a manganese-cofactored superoxide dismutase. Infection and Immunity. 1992;60(2):328-36. 
57. Gardner PR. Superoxide production by the mycobacterial and pseudomonad quinoid pigments phthiocol and pyocyanine in human lung cells. Archives of Biochemistry and Biophysics. 1996;333(1):267-74.

58. Meirelles LA, Newman DK. Both toxic and beneficial effects of pyocyanin contribute to the lifecycle of Pseudomonas aeruginosa. Molecular Microbiology. 2018;110(6):995-1010.

59. Das T, Manefield M. Pyocyanin promotes extracellular DNA release in Pseudomonas aeruginosa. PLOS One. 2012;7(10):e46718.

60. Hassett DJ, Ma J-F, Elkins JG, McDermott TR, Ochsner UA, West SEH, et al. Quorum sensing in Pseudomonas aeruginosa controls expression of catalase and superoxide dismutase genes and mediates biofilm susceptibility to hydrogen peroxide. Molecular Microbiology. 1999;34(5):1082-93.

61. García-Contreras R, Nuñez-López L, Jasso-Chávez R, Kwan BW, Belmont JA, RangelVega A, et al. Quorum sensing enhancement of the stress response promotes resistance to quorum quenching and prevents social cheating. The ISME Journal. 2015;9(1):115-25.

62. Pfluger-Grau K, Gorke B. Regulatory roles of the bacterial nitrogen-related phosphotransferase system. Trends Microbiol. 2010;18(5):205-14.

63. Velazquez F, Pfluger K, Cases I, De Eugenio LI, de Lorenzo V. The phosphotransferase system formed by PtsP, PtsO, and PtsN proteins controls production of polyhydroxyalkanoates in Pseudomonas putida. Journal of bacteriology. 2007;189(12):4529-33.

64. Tan MW, Rahme LG, Sternberg JA, Tompkins RG, Ausubel FM. Pseudomonas aeruginosa killing of Caenorhabditis elegans used to identify P. aeruginosa virulence factors. Proceedings of the National Academy of Sciences of the United States of America. 1999;96(5):2408-13.

65. Schurek KN, Marr AK, Taylor PK, Wiegand I, Semenec L, Khaira BK, et al. Novel genetic determinants of low-level aminoglycoside resistance in Pseudomonas aeruginosa. Antimicrobial agents and chemotherapy. 2008;52(12):4213.

66. Scribner MR, Santos-Lopez A, Marshall CW, Deitrick C, Cooper VS. Parallel evolution of tobramycin resistance across species and environments. mBio. 2020;11(3). 
638 67. Poole K. Aminoglycoside resistance in Pseudomonas aeruginosa. Antimicrob Agents 639 Chemother. 2005;49(2):479-87.

640 68. Li X-Z, Poole K, Nikaido H. Contributions of MexAB-OprM and an EmrE homolog to 641 intrinsic resistance of Pseudomonas aeruginosa to aminoglycosides and dyes. Antimicrob Agents 642 Chemother. 2003;47(1):27-33.

643 69. Aires JR, Köhler T, Nikaido H, Plésiat P. Involvement of an active efflux system in the 644 natural resistance of Pseudomonas aeruginosa to aminoglycosides. Antimicrob Agents 645 Chemother. 1999;43(11):2624-28.

646 70. Masuda N, Sakagawa E, Ohya S GN, Tsujimoto H, Nishino T. Contribution of the 647 MexX-MexY-oprM efflux system to intrinsic resistance in Pseudomonas aeruginosa. Antimicrob 648 Agents Chemother. 2000;44(9):2242-6.

649 71. Zhang L, Mah TF. Involvement of a novel efflux system in biofilm-specific resistance to 650 antibiotics. J Bacteriol. 2008;190(13):4447-52.

651 72. Scholz RL, Greenberg EP. Positive autoregulation of an acyl-homoserine lactone 652 quorum-sensing circuit synchronizes the population response. mBio. 2017;8(4).

653 73. El Meouche I, Dunlop MJ. Heterogeneity in efflux pump expression predisposes 654 antibiotic-resistant cells to mutation. Science. 2018;362(6415):686-90. 


\section{FIGURE LEGENDS}

658

659

660

661

662

663

664

665

666

667

668

669

670

671

672

673

674

675

676

677 represent the standard deviation.
Fig. 1. lasR contributes to tobramycin resistance in planktonically-grown $P$. aeruginosa PA14.

A. The minimum inhibitory concentration (MIC) of tobramycin was determined for each of the strains indicated as described in Materials and Methods. 30C12-HSL was added before inoculation where indicated $(10 \mu \mathrm{M}$ final). Statistical analysis by one-way ANOVA and Dunnett's multiple comparisons test with wild type: *** $\mathrm{p}<0.001$, $* * * * \mathrm{p}<0.0001$. B. Cells recovered following tobramycin treatment $\left(1.1 \mu \mathrm{g} \mathrm{ml}^{-1}\right)$. Cells were treated as in part A and the surviving cells were enumerated by serial dilution and plating following treatment. For both experiments, the values shown represent the average of three independent experiments and the error bars represent the standard deviation.

Fig. 2. Tobramycin suppresses the emergence of lasR-mutant cheaters. P. aeruginosa populations were transferred daily in $1 \%$ casein broth for 32 days, and cheaters were enumerated every 4 days by patching as described in Materials and Methods. For each experiment, one culture was initially started with no tobramycin, and after $72 \mathrm{hr}$ split into three cultures propagated in one of three conditions; i) with no tobramycin $\left(-\mathrm{O}^{-}\right)$, ii) with tobramycin added at $0.6 \mu \mathrm{g} \mathrm{ml}^{-1}(-)$, iii) with tobramycin added at $0.6 \mu \mathrm{g} \mathrm{ml}^{-1}$ initially, and increased by $50 \%$ every 4 days to a final concentration of $7.1 \mu \mathrm{g} \mathrm{ml}^{-1}(\Delta)$. For conditions ii and iii, tobramycin was added every other day just after transfer to fresh medium. The detection level of cheaters was $1 \%$. The values shown represent the average of three independent experiments and the error bars 678 
679 Fig. 3. Effects of $p t s P$ inactivation on LasR activity and pyocyanin production. A. Activity of 680 LasR. P. aeruginosa strains were electroporated with a plasmid containing a LasR-responsive 681 GFP reporter (pBS351). Reported values are fluorescence normalized to culture density at $18 \mathrm{~h}$

682 of growth. B. Pyocyanin production. Cultures were inoculated into pyocyanin-producing media, 683 grown for $18 \mathrm{~h}$, and extracted before quantifying pyocyanin as described in Materials and 684 Methods. In all cases, reported values are $\mu \mathrm{g} / \mathrm{ml}$ pyocyanin normalized to culture density at the 685 time of measurement. Strains carried either the empty CTX-1 cassette (CTX), or the CTX-1-ptsP cassette, inserted at the neutral $a t t B$ site in the genome. The values represent the average of three independent experiments and the error bars represent the standard deviation. Statistical analysis by one-way ANOVA and Dunnett's multiple comparisons test with wild type: ** $\mathrm{p}<0.01$, *** $\mathrm{p}<0.001, * * * * \mathrm{p}<0.0001$

Fig. 4. pts $P$ inactivation suppresses cheaters. P. aeruginosa populations were transferred daily in $1 \%$ casein broth for 32 days, and cheaters were enumerated every 4 days by patching as described in Materials and Methods. Values shown indicate the number of days until lasRmutated cheaters emerge in A) populations of wild type PA14 (WT) and PA14 variants (T1, T2 and T3) from tobramycin-evolved populations and B) WT or the $\Delta p t s P$ mutant carrying either

696 the empty CTX-1 cassette (CTX), or the CTX-1-ptsP (CTX-ptsP) cassette, inserted at the neutral $697 a t t B$ site in the genome. Cheater emergence is defined as when las $R$ mutants reach a frequency in 698 the population of $8 \%$, at which point it was generally observed that cheater frequencies 699 continued increasing rather than decreasing or remaining constant. Full data sets of cheater 700 frequency and total populations are shown in Fig. S5. Values represent the average of three 701 independent experiments and the error bars represent the standard deviation. Statistical analysis 
702

703

704

705

706

707

708

709

710

711

712

713

714

715

716

717

718

719

720

721

722

723

724

by one-way ANOVA and Dunnett's multiple comparisons test with wild type for A; and by oneway ANOVA and Tukey's multiple comparisons test for B: **** $\mathrm{p}<0.0001$.

Fig. 5. $\triangle p t s P$ mutant cultures has antimicrobial activity against las $R$ mutants. Final cell densities of $P$. aeruginosa strains treated for $24 \mathrm{~h}$ with filtered fluid from $1 \%$ casein-grown cultures of untreated (black), wild-type PA14 (light grey), or the ptsP mutant (dark grey). The initial cell density of the treated cultures was $5-7 \times 10^{5}$ cells $\mathrm{ml}^{-1}$. The values represent the average of three independent experiments and the error bars represent the standard deviation. Statistical analysis by two-way ANOVA and Dunnett's multiple comparison test with untreated for each strain. * $\mathrm{p}<0.05, * * \mathrm{p}<0.01$

Fig. 6. $\Delta p t s P$ mutants police cheaters using pyocyanin. A. Pyocyanin biosynthesis steps. The products of the phzA1-G1 (phz1) and phzA2-G2 (phz2) gene clusters synthesize phenazine-1carboxylic acid (PCA) from chorismate. PhzM and PhzH convert PCA into 5-methylphenazine 1-carboxylic acid (5-Me-PCA) or phenazine-1-carboxamide (PCN), respectively. PhzS converts PCA into pyocyanin. B. Final cell densities of $\Delta p t s P, \Delta l a s R$ cells treated with filtered fluid from $1 \%$ casein-grown cultures of a $\Delta p t s P$ mutant or $\Delta p t s P$ with the entire phz1 and phz2 operons deleted $(\Delta p h z 1-2)$ or with $p h z M, p h z S$, or $p h z H$ deleted. The initial cell density of the treated cultures was 5-7 X $10^{5}$ cells $\mathrm{ml}^{-1}$. Data are the average of three independent experiments with standard deviation. Statistical analysis by one-way ANOVA and Tukey's multiple comparisons test with $\Delta p t s P,{ }^{*} \mathrm{p}<0.05$. C. Final cell densities of wild-type PA14 (WT) or the $\Delta p t s P, \Delta l a s R$ mutant treated with 0 or $10 \mu \mathrm{g} \mathrm{ml}^{-1}$ pyocyanin and filtered fluid from $1 \%$ casein-grown $\Delta p t s P$ $\triangle p h z S$ cultures. The initial cell density of treated cultures was $4-6 \times 10^{5} \mathrm{CFU} \mathrm{ml}^{-1}$. Data are the 
725 average of four independent experiments with standard deviations. Statistical analysis by two-

726 way ANOVA and multiple comparisons test with no-pyocyanin control, * $\mathrm{p}<0.05$. D. Cheater

727 suppression during competition. Competitions were inoculated with the las $R$ mutant and each

728 cooperator strain at an initial ratio of 1:99 (cheater:cooperator) in $1 \%$ casein broth and

729 transferred to fresh medium daily for 3 days. On day 3, cheaters were enumerated by patching as

730 described in Materials and Methods. Each data point represents an independent experiment. The

731 horizontal line represents the mean and the vertical line represents the standard deviation of all

732 the experiments in each set. Statistical analysis by one-way ANOVA with Tukey's multiple

733 comparisons test of each set with the wild-type cooperator experiments: $* * \mathrm{p}<0.01, * * * \mathrm{p}<0.001$.

734 Total population densities of each experiment are shown in Fig. S6.

735

736

737

738

739

740

741

742 


\section{TABLES}

744 Table 1. Mutations in variants from populations passed with $0.6-7.1 \mu \mathrm{g} \mathrm{ml}^{-1}$ tobramycin.

\begin{tabular}{c|c|c|l}
\hline \multirow{2}{*}{ Variant } & \multicolumn{3}{|c}{ Gene mutation $^{\text {a }}$} \\
\cline { 2 - 4 } & $p t s P$ & fusA1 & \multicolumn{1}{|c}{ others } \\
\hline $\mathrm{T} 1$ & $1547 \mathrm{~T}$ & $\mathrm{G} 61 \mathrm{~A}$ & $p m r B$, mdpA \\
$\mathrm{T} 2$ & $841 \Delta \mathrm{C}$ & $\mathrm{ND}^{\mathrm{b}}$ & $\operatorname{mexZ}$, rrf2, psdR, PA14_RS15595 \\
$\mathrm{T} 3$ & $1547 \mathrm{~T}$ & $\mathrm{G} 61 \mathrm{~A}$ & $\operatorname{dppA3,ostA}$ \\
\hline
\end{tabular}

${ }^{\mathrm{a}}$ Mutations are given by nucleotide location

$746 \quad{ }^{\mathrm{b}} \mathrm{ND}$ : not detected 

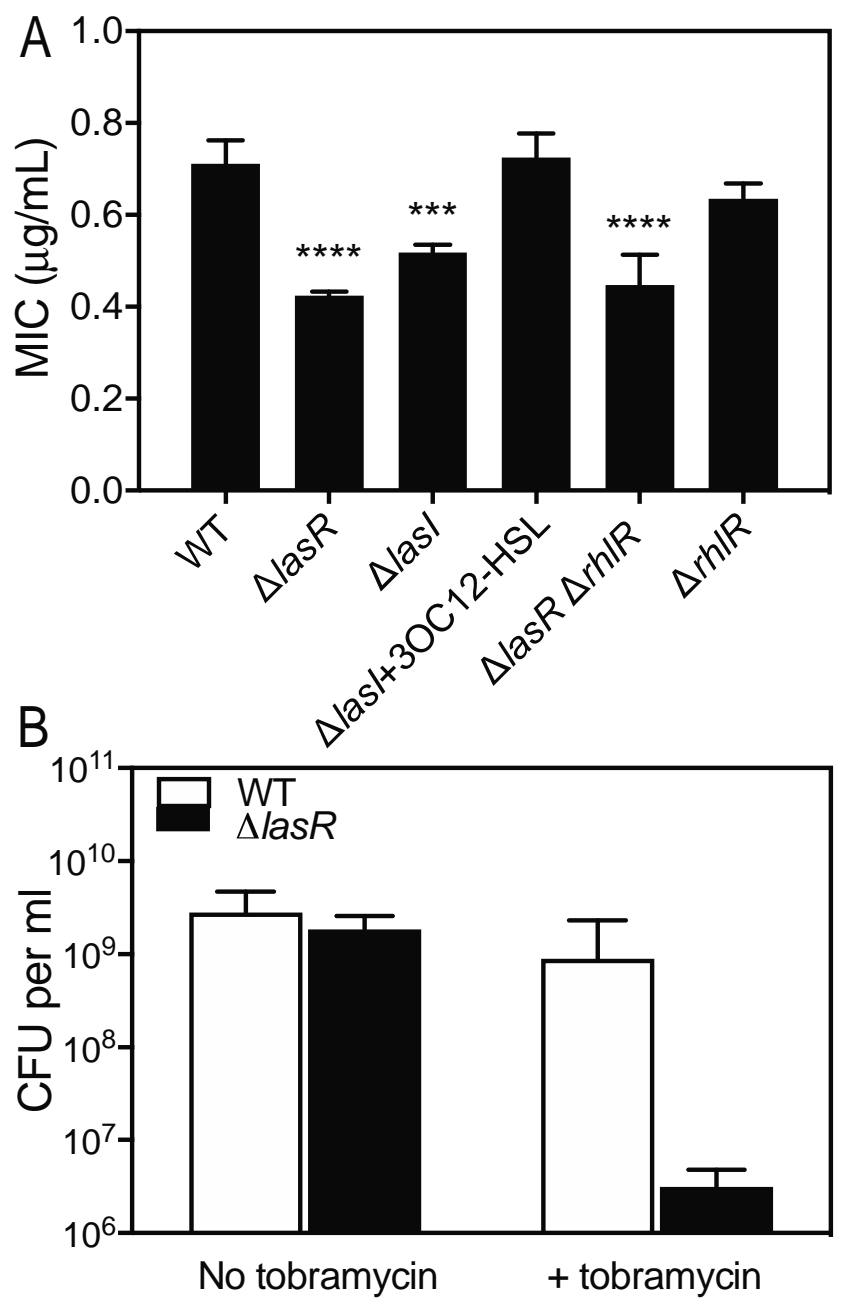

Fig. 1 


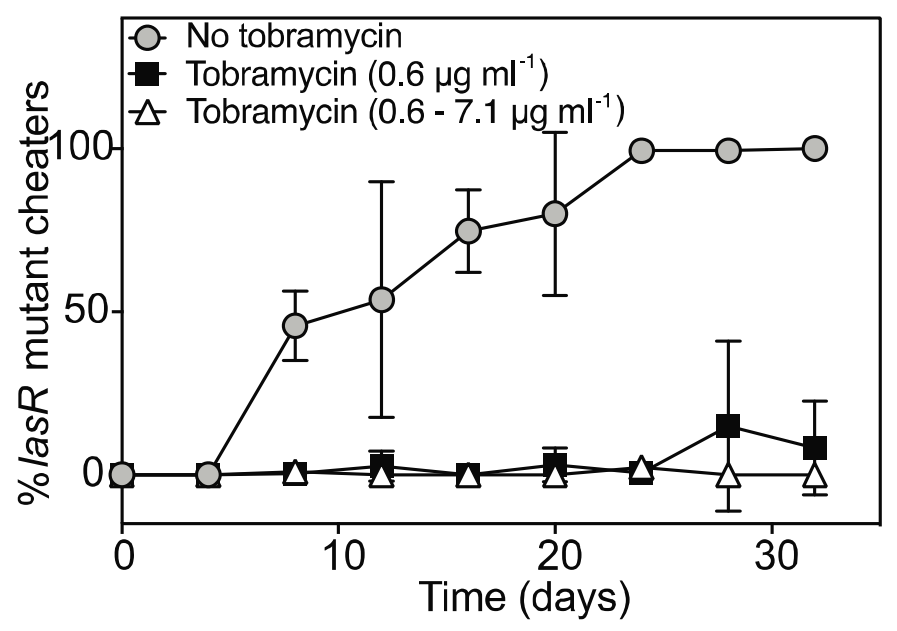

753 Fig. 2.

754

755

756

757

758

759

760

761

762

763

764 

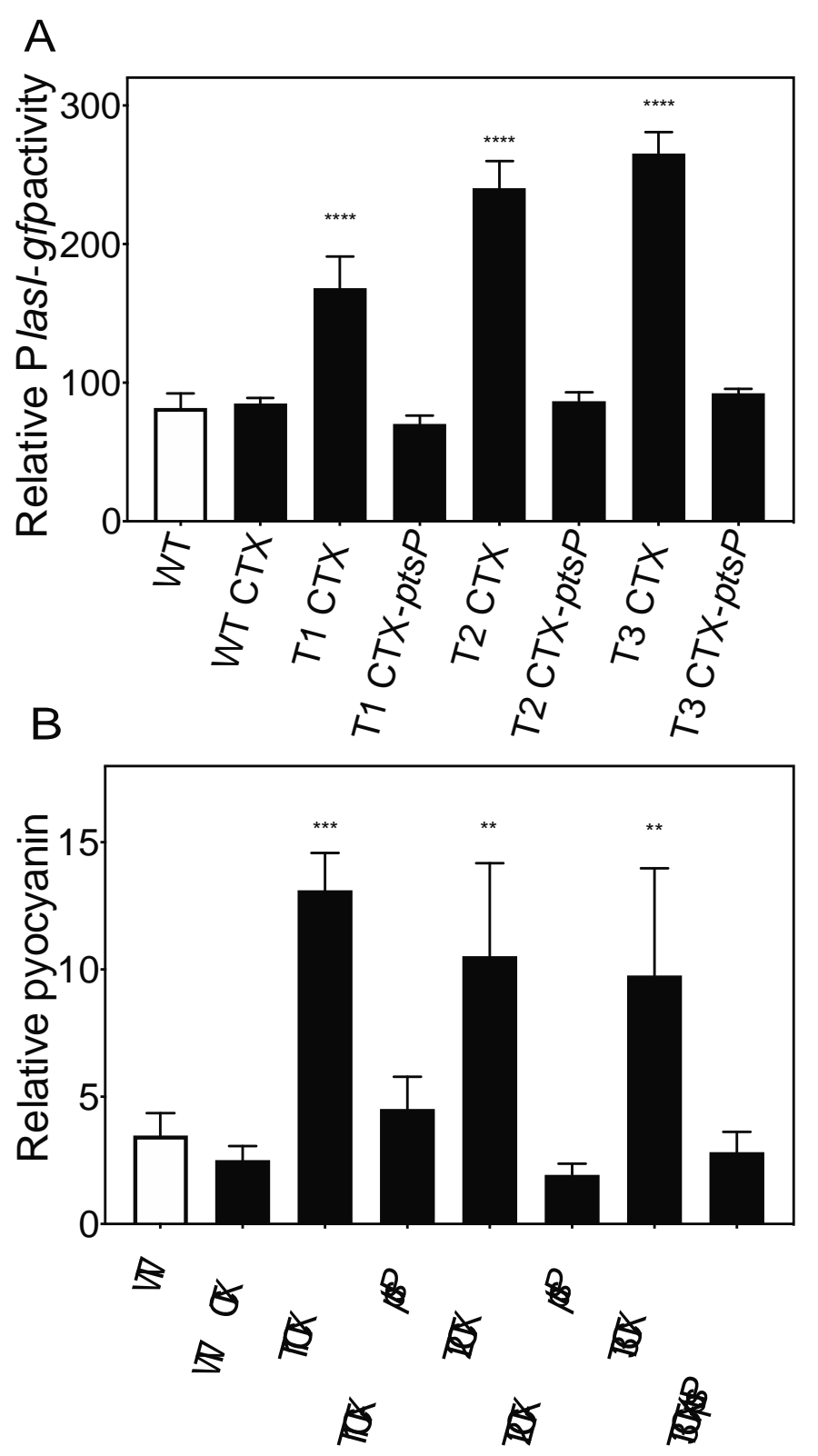

Fig. 3 
A

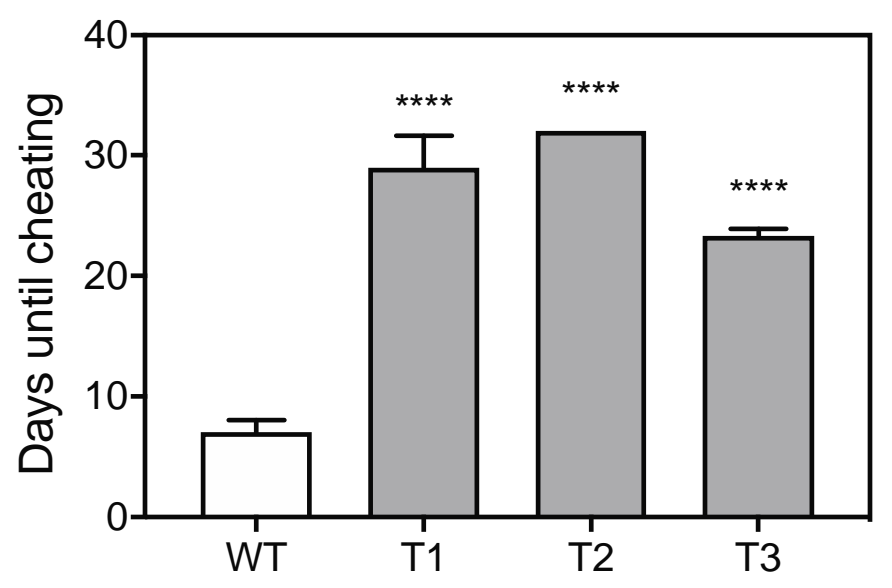

B

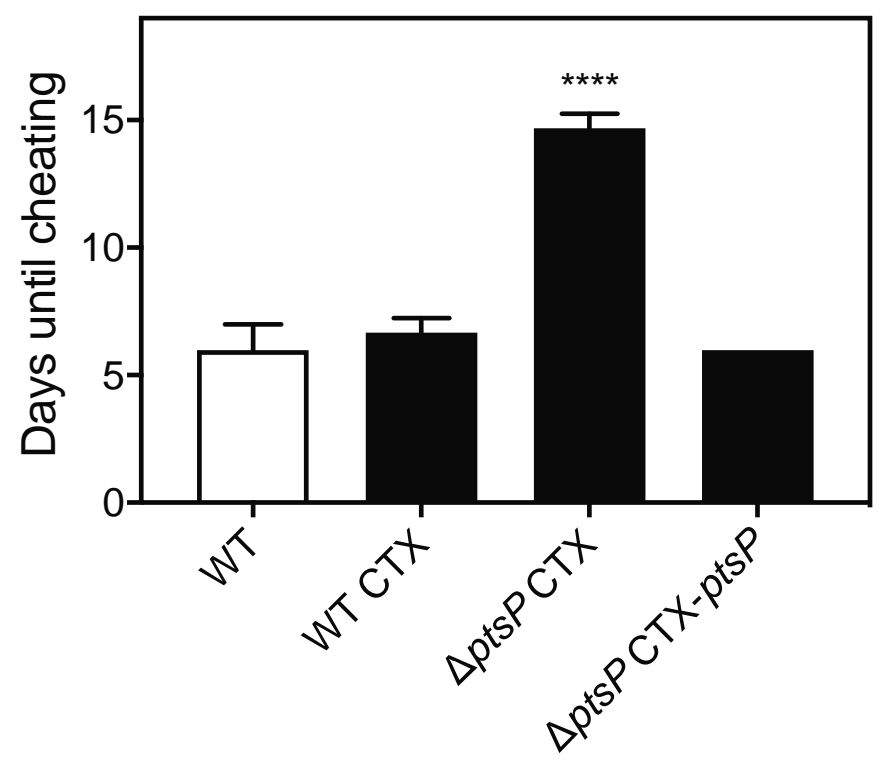

Fig. 4 


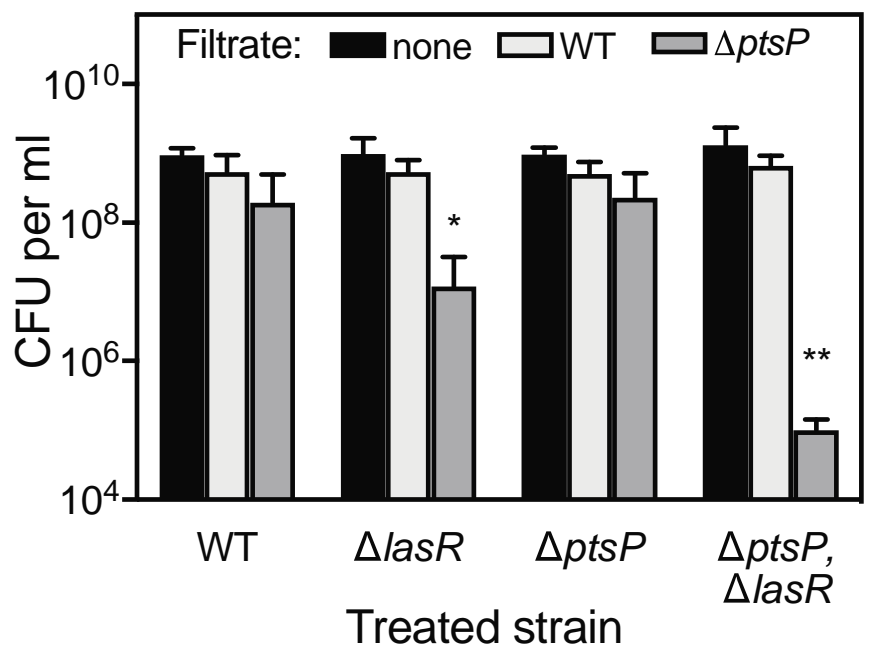

$778 \quad$ Fig. 5

779 


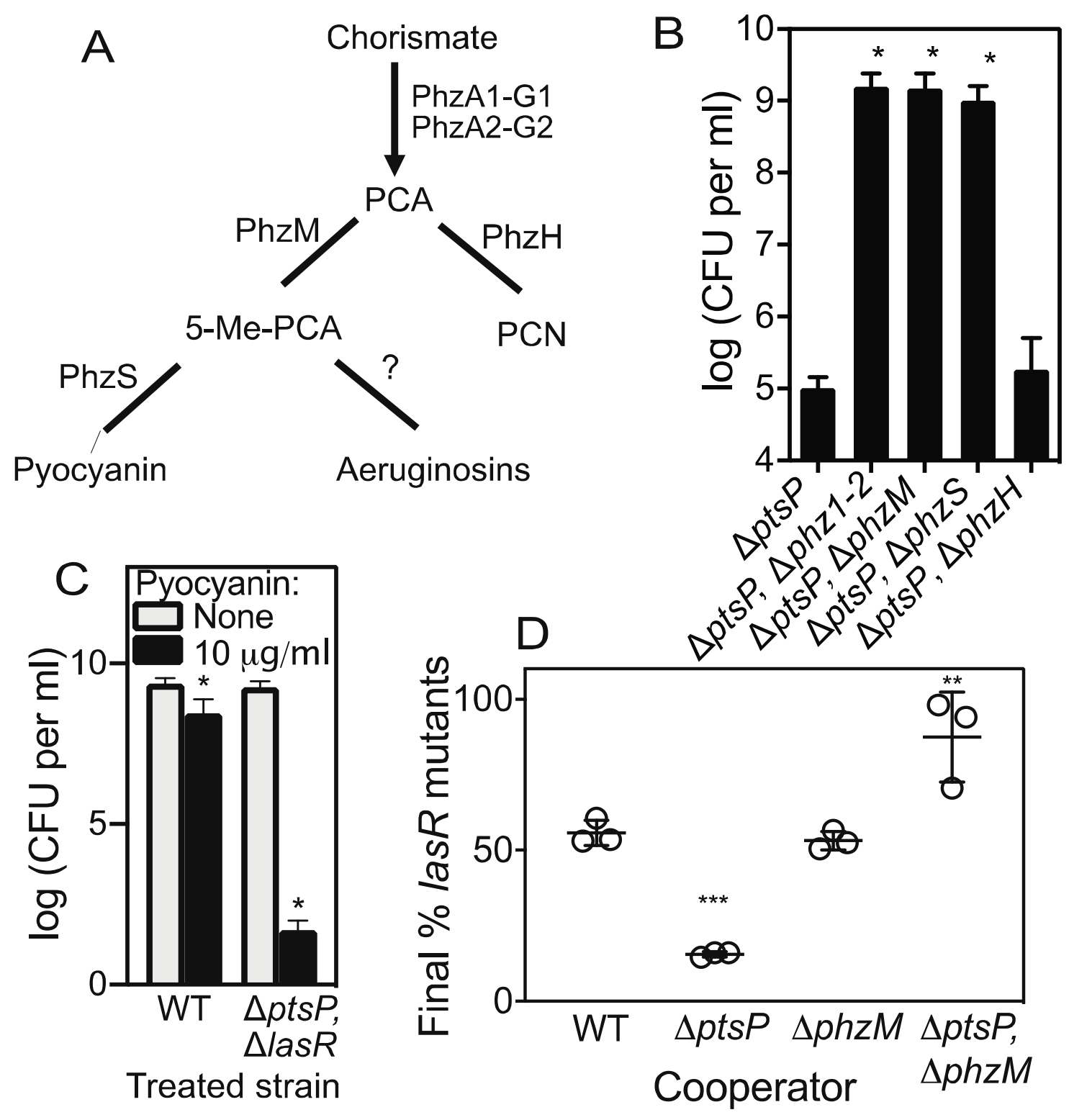

Fig. 6 